\title{
Front and Back Movement Analysis of a Triangle-Structured Three-Wheeled Omnidirectional Mobile Robot by Varying the Angles between Two Selected Wheels
}

\author{
A. P. Mohanraj, ${ }^{1}$ A. Elango, ${ }^{2}$ and Mutra Chanakya Reddy ${ }^{1}$ \\ ${ }^{1}$ School of Mechanical Engineering, SASTRA University, Thanjavur, Tamil Nadu 613401, India \\ ${ }^{2}$ Department of Mechanical Engineering, ACCET, Karaikudi, Tamil Nadu 630004, India \\ Correspondence should be addressed to A. P. Mohanraj; apmohanraj@mech.sastra.edu
}

Received 24 June 2015; Accepted 14 January 2016

Academic Editor: Andrea Vania

Copyright (C) 2016 A. P. Mohanraj et al. This is an open access article distributed under the Creative Commons Attribution License, which permits unrestricted use, distribution, and reproduction in any medium, provided the original work is properly cited.

Omnidirectional robots can move in all directions without steering their wheels and it can rotate clockwise and counterclockwise with reference to their axis. In this paper, we focused only on front and back movement, to analyse the square- and trianglestructured omnidirectional robot movements. An omnidirectional mobile robot shows different performances with the different number of wheels and the omnidirectional mobile robot's chassis design. Research is going on in this field to improve the accurate movement capability of omnidirectional mobile robots. This paper presents a design of a unique device of Angle Variable Chassis (AVC) for linear movement analysis of a three-wheeled omnidirectional mobile robot (TWOMR), at various angles $(\theta)$ between the wheels. Basic mobility algorithm is developed by varying the angles between the two selected omnidirectional wheels in TWOMR. The experiment is carried out by varying the angles $\left(\theta=30^{\circ}, 45^{\circ}, 60^{\circ}, 90^{\circ}\right.$, and $\left.120^{\circ}\right)$ between the two selected omniwheels and analysing the movement of TWOMR in forward direction and reverse direction on a smooth cement surface. Respectively, it is compared to itself for various angles $(\theta)$, to get its advantages and weaknesses. The conclusion of the paper provides effective movement of TWOMR at a particular angle $(\theta)$ and also the application of TWOMR in different situations.

\section{Introduction}

Omnidirectional mobile robots are useful in a large variety of indoor service applications such as industrial, medical, and domestic purposes. Omnidirectional mobile robots provide high mobility compared to the more common car-like robots. Some of the omnidirectional mobile robot platforms are designed for use in the highway maintenance and congested environment [1]. The omnidirectional chassis has many forms; the three-wheel and four-wheel schemes are the most familiar. The number of wheels and the layout can both affect the performances of the chassis [2-4]. There have been several works on the applications of using omnidirectional mobile robots $[5,6]$, the development of three special wheeled structures, and also the isotropy analysis of the mobile robots and kinematic analysis. There have been several works on the impact of the number of wheels and layouts to the performances of omnidirectional chassis [7-9]. There are studies on motion planning algorithms of an omnidirectional mobile robot, which helps us to understand the movement of an omnidirectional mobile robot [10-12]. Caster drive mechanism for the omnidirectional mobile robot platforms is also attractive aspect [13-20].

The angle variations between those two wheels will enable us to analyse the sideways movement of triangular structure and crossway movements of square structure omnidirectional robot. In this paper, the forward and reverse movement analysis and deflections caused due to the various angles between two wheels, like $\theta=30^{\circ}, 45^{\circ}, 60^{\circ}, 90^{\circ}$, and $120^{\circ}$, were experimentally analysed. Therefore, the paper attempts to analyse how the angle variations between the two wheels 
TABLE 1: Specifications of omniwheel.

\begin{tabular}{lc}
\hline Wheel & Specifications \\
\hline Outer diameter & $100 \mathrm{~mm}$ \\
Bore (hole) diameter & $13 \mathrm{~mm}$ with $3 \mathrm{~mm}$ key \\
Wheel thickness & $38 \mathrm{~mm}$ \\
Number of rollers & 2 rows and 9 rollers each \\
Weight & $30 \mathrm{~g}$ \\
Load capacity & $20 \mathrm{~kg}$ \\
\hline
\end{tabular}

TABLE 2: Specifications of DC motor.

\begin{tabular}{lc}
\hline Motor & Specifications \\
\hline Speed & $45 \mathrm{rpm}$ \\
Torque & $0.1961 \mathrm{Nm}$ \\
Voltage & $12 \mathrm{~V}$ \\
\hline
\end{tabular}

are giving impact on the movement of omnidirectional mobile robots. The theoretical analysis and experimental tests were conducted in order to demonstrate the performance of TWOMR.

\section{The Omnidirectional Mobile Robot Platform}

The double row omnidirectional wheel is shown in Figure 1, and specifications of omniwheel and DC motor are mentioned in Tables 1 and 2, respectively. It can move forward, backward, and sideways also by using the rotation of the rollers. The Pro-E design and the prototype of three-wheeled omnidirectional mobile robot (TWOMR) are shown in Figures 3 and 4 .

To analyse different angles of TWOMR, a unique device, Angle Variable Chassis (AVC), is designed as shown in Figure 2; it is having two "side bodies," having $180 \mathrm{~mm}$ length for a $100 \mathrm{~mm}$ diameter omniwheel. Two side extensions are there in each "side body" with $6 \mathrm{~mm}$ diameter hole. The front connector is designed as $90 \mathrm{~mm}$ in length and $20 \mathrm{~mm}$ in width. It is having 2 holes in $6 \mathrm{~mm}$ diameter. The back connector is having a length of $400 \mathrm{~mm}$ and width of $40 \mathrm{~mm}$.

A $12 \mathrm{~mm}$ slot has been created for nearly $160 \mathrm{~mm}$ long, to connect with the back side of the body. All the structures had been fabricated in $2 \mathrm{~mm}$ thick mild steel plate, for its stiffness. "Side body" and front and back connector plates were connected by bolt, nut, and some washers. By adjusting the "chassis side bodies" of the "Angle Variable Chassis" (AVC), the angle between the wheels can be varied.

The proposed Pro-E design and prototype of TWOMR are shown in Figures 3 and 4, respectively. The three-wheeled omnidirectional mobile robots are capable of achieving 3 DOF motions by driving three independent actuators. So the mobile robot used in this research is a holonomic omnidirectional mobile robot. The chassis is designed in such

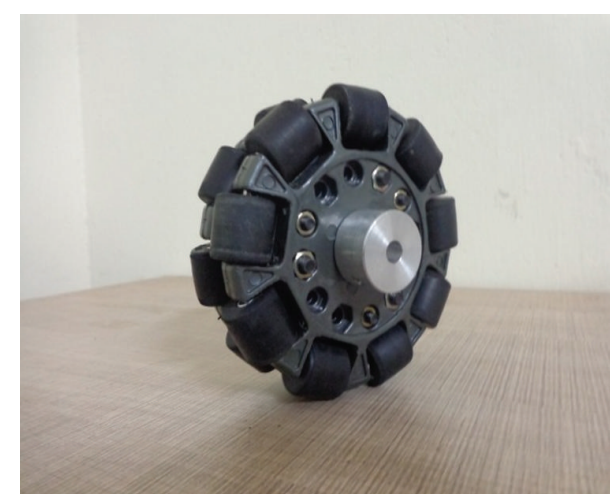

Figure 1: Omnidirectional wheel.

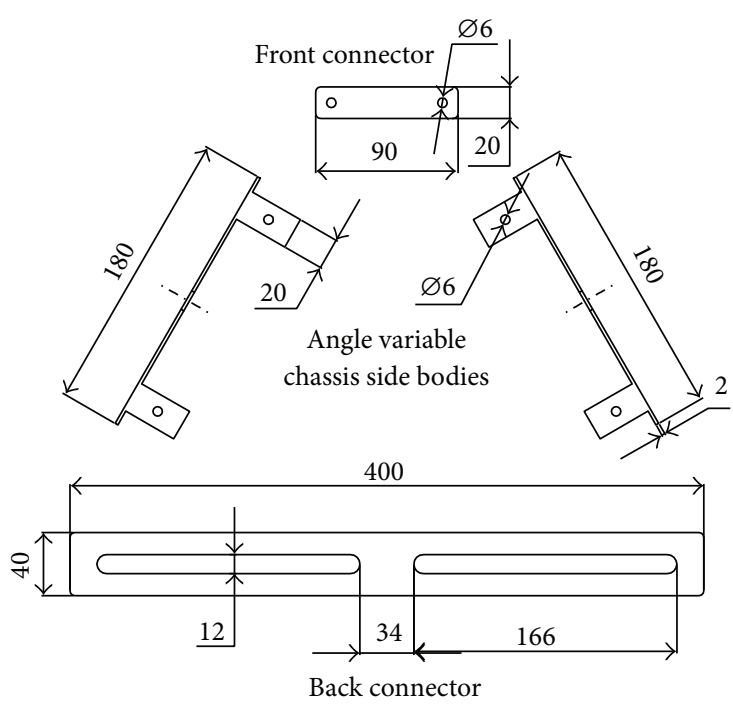

Figure 2: Angle Variable Chassis (AVC) side body and front and back connectors with dimensions (all dimensions are in $\mathrm{mm}$ ).

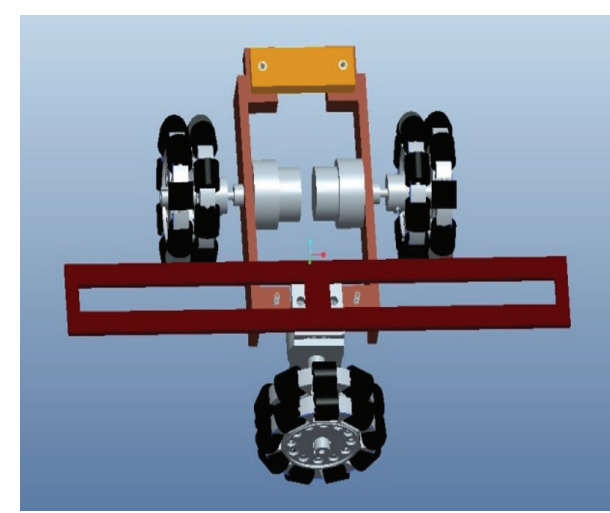

Figure 3: Pro-E design of TWOMR.

a way that the angles between omniwheel 1 and omniwheel 2 from Figure 4 can be maintained from $10^{\circ}$ to $170^{\circ}$.

Hence, the experiments are carried out by varying the angles between omniwheel 1 and omniwheel 2, neglecting omniwheel 3. Each wheel is fixed with an independent DC motor actuator and each DC motor actuator is controlled 


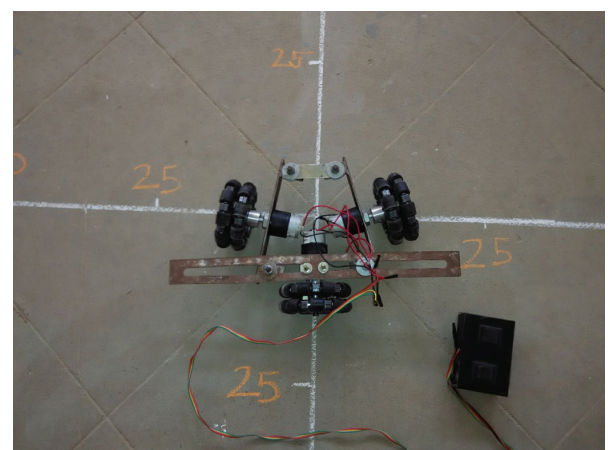

FIgURE 4: Prototype of TWOMR.

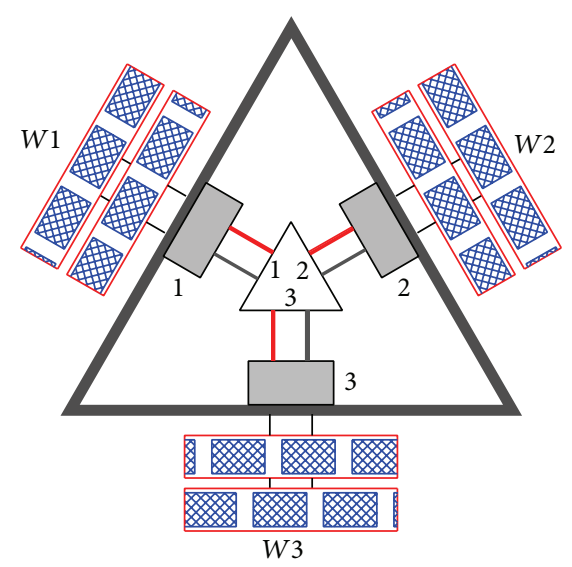

Figure 5: The circuit diagram of TWOMR (1, 2, and 3 are DC motors and $W 1, W 2$, and $W 3$ are omniwheels).

by a remote controller. The circuit diagram is shown in Figure 5. Only omniwheel 1 and omniwheel 2 are used for the movement of the mobile robot; omniwheel 3 is used to provide the robot stability but not for the movement of the mobile robot. Omniwheels 1 and 2 should move with the same speed and each omniwheel has the capability to drive independently. Each omniwheel driven by a DC motor consists of eighteen orthogonal rollers and provides twodirectional movements. With a proper mechanical design of chassis, the TWOMR can be able to move in forward and reverse direction by varying the angles between omniwheel 1 and omniwheel 2 from $\theta=10^{\circ}$ to $170^{\circ}$.

This Angle Variable Chassis (AVC) is designed to vary the angles of triangle-structured omnidirectional mobile robot which is having $60^{\circ}$ in all sides (equilateral triangle). The experiments were conducted for $10^{\circ}$ to $170^{\circ}$; in this paper, the experimental tests are shown for some important angles used in a triangle, like $30^{\circ}, 45^{\circ}, 60^{\circ}, 90^{\circ}$, and $120^{\circ}$, because when the angle is $0^{\circ}$, omniwheel 1 and omniwheel 2 are parallel to each other. The omniwheels almost acted like a normal wheel. When the angles were increased from $0^{\circ}$, then two omniwheels were creating resultant force.

For angles between $0^{\circ}$ and $30^{\circ}$, the TWOMR did not show much deviation. When it is reached at the range of

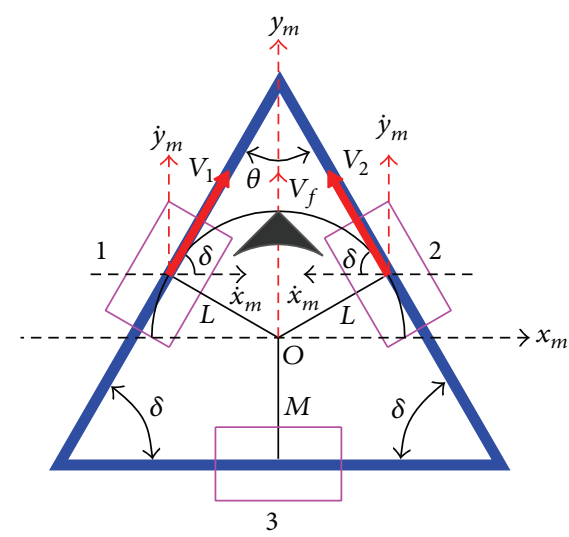

FiguRE 6: Forward movement schematic of chassis.

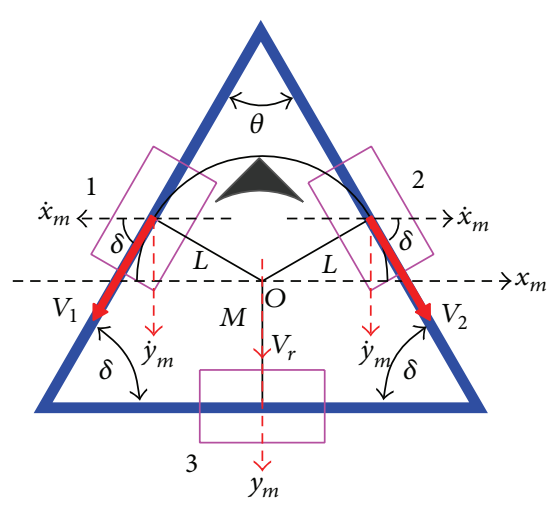

Figure 7: Reverse movement schematic of chassis.

$30^{\circ}$, it has shown some deviations. When the angles are beyond $120^{\circ}$ to $180^{\circ}$, omniwheel 1 and omniwheel 2 could not create the desired resultant force for its movement. When the angles have been increased beyond $120^{\circ}$ to $180^{\circ}$, the two omniwheels are creating almost equal and opposite forces, so that the TWOMR is struggling to create the front and back movements. So, in this paper, the experimental analysis has been shown up to $120^{\circ}$.

\section{Kinematic Model of the TWOMR}

The schematic of the chassis is shown in Figures 6 and 7, where a full "black" triangle indicates the forehead of the robot. The length between omniwheels 1 and 2 and centre $O$ is $L$ and the length between omniwheel 3 and centre $O$ is $M$.

The lengths $L$ and $M$ change according to angle $\theta$ between omniwheel 1 and omniwheel 2 . We define the fact that velocity $V_{1}$ and velocity $V_{2}$ of omniwheel 1 and omniwheel 2 , respectively, are positive when the robot rotates counterclockwise. As shown in Figures 6 and 7, the mobile coordinate frame $\left[x_{m}, y_{m}\right]$ is located at the centre of gravity of the robot. The two front wheels ( 1 and 2 ) are offset from " $x_{m}$ " by fixed angle $\delta$. Angle $\delta$ changes according to angle $\theta$ between omniwheel 1 and omniwheel 2.

According to the schematic of the chassis with three omniwheels, we can derive the kinematics model of the 
TWOMR using trigonometry and geometry. Assume the radius of each omniwheel is $R$ and its rotating rate is $\omega_{i}$. We can derive the velocity of each omniwheel by

$$
V_{i}=R \omega_{i}, \quad i=1,2,
$$

where $i$ denotes the $i$ th omniwheel. When the TWOMR moves in the forward direction at velocity $V_{f}$, velocities $V_{1}$ and $V_{2}$ can be derived as follows:

$$
\begin{aligned}
& V_{1}=R \omega_{1}=+\cos (\delta) \dot{x}+\sin (\delta) \dot{y}, \\
& V_{2}=R \omega_{2}=-\cos (\delta) \dot{x}+\sin (\delta) \dot{y},
\end{aligned}
$$

where $\dot{x}$ and $\dot{y}$ denote the velocity of the direction of $x_{m}$ and $y_{m}$, respectively. Equations (2) can be rewritten as

$$
\left[\begin{array}{l}
V_{1} \\
V_{2}
\end{array}\right]=\left[\begin{array}{l}
R \omega_{1} \\
R \omega_{2}
\end{array}\right]=\left[\begin{array}{cc}
\cos (\delta) & \sin (\delta) \\
-\cos (\delta) & \sin (\delta)
\end{array}\right]\left[\begin{array}{l}
\dot{x} \\
\dot{y}
\end{array}\right] .
$$

The forward resultant velocity $\left(V_{f}\right)$ can be written as

$$
V_{f}=V_{1}+V_{2}=2 \sin (\delta) \dot{y} .
$$

Similarly, when the TWOMR moves in the reverse direction at velocity $V_{r}$, velocities $V_{1}$ and $V_{2}$ can be derived as follows:

$$
\begin{aligned}
& V_{1}=R \omega_{1}=-\cos (\delta) \dot{x}-\sin (\delta) \dot{y}, \\
& V_{2}=R \omega_{2}=+\cos (\delta) \dot{x}-\sin (\delta) \dot{y},
\end{aligned}
$$

where $\dot{x}$ and $\dot{y}$ denote the velocity of the direction of $x_{m}$ and $y_{m}$, respectively.

Equations (5) can be rewritten as

$$
\left[\begin{array}{l}
V_{1} \\
V_{2}
\end{array}\right]=\left[\begin{array}{l}
R \omega_{1} \\
R \omega_{2}
\end{array}\right]=\left[\begin{array}{cc}
-\cos (\delta) & -\sin (\delta) \\
\cos (\delta) & -\sin (\delta)
\end{array}\right]\left[\begin{array}{l}
\dot{x} \\
\dot{y}
\end{array}\right] .
$$

The reverse resultant velocity $\left(V_{r}\right)$ can be written as

$$
V_{r}=V_{1}+V_{2}=-2 \sin (\delta) \dot{y},
$$

where negative sign indicates reverse direction.

\section{Theoretical Analysis of TWOMR}

In the theoretical analysis, some assumptions are made; for example, there are no effect of friction on TWOMR, no effect of omniwheel rollers on omniwheels, and no errors in the design and manufacturing process.

From Figures 6 and 7, the schematic of the chassis and from (4) and (7), the resultant velocity forward and reverse respectively; Table 3 shows the relations.

Table 3 clearly shows that there is an impact of angle $(\theta)$ on the resultant velocity in both forward $\left(V_{f}\right)$ and reverse $\left(V_{r}\right)$ direction. As we can see, increasing the angle $(\theta)$ from $30^{\circ}$ to $120^{\circ}$, there is a decrease in the angle $(\delta)$ from $75^{\circ}$ to $30^{\circ}$; the sine value decreases from $75^{\circ}$ to $30^{\circ}$. Hence, at an angle $\left(\theta=30^{\circ}\right)$, the sine value at $75^{\circ}$ is maximum, so maximum velocity is obtained in both forward and reverse direction.
TABLE 3: Relation between angles $\theta$ and $\delta$.

\begin{tabular}{lccc}
\hline S. number & Angle $(\theta)$ & Angle $(\delta)$ & Resultant velocity \\
\hline 1 & $30^{\circ}$ & $75^{\circ}$ & $\pm 2 \sin \left(75^{\circ}\right) \dot{y}$ \\
2 & $45^{\circ}$ & $67.5^{\circ}$ & $\pm 2 \sin \left(67.5^{\circ}\right) \dot{y}$ \\
3 & $60^{\circ}$ & $60^{\circ}$ & $\pm 2 \sin \left(60^{\circ}\right) \dot{y}$ \\
4 & $90^{\circ}$ & $45^{\circ}$ & $\pm 2 \sin \left(45^{\circ}\right) \dot{y}$ \\
5 & $120^{\circ}$ & $30^{\circ}$ & $\pm 2 \sin \left(30^{\circ}\right) \dot{y}$ \\
\hline
\end{tabular}

The positive sign corresponds to the forward velocity $V_{f}$ and the negative sign to the reverse velocity $V_{r}$.

At angle $\left(\theta=120^{\circ}\right)$, the sine value at $30^{\circ}$ is minimum, so minimum velocity is obtained in both forward and reverse direction. As a result, if the angle $(\theta)$ between omniwheel 1 and omniwheel 2 increases, there will be a decrease in the resultant velocity in both forward $\left(V_{f}\right)$ and reverse $\left(V_{r}\right)$ direction and vice versa.

Theoretically, there will not be any deflections or deviations occurring at angles $\left(\theta=30^{\circ}, 45^{\circ}, 60^{\circ}, 90^{\circ}\right.$, and $\left.120^{\circ}\right)$ in both forward and reverse direction. The omnidirectional mobile robot has to travel in straight paths in both forward and reverse direction.

Figures 6 and 7 show the forward and reverse resultants, respectively, which are straight paths, and omnidirectional mobile robot is supposed to travel in straight paths without any small deflections or deviations in both forward and reverse direction at all angles $\left(\theta=30^{\circ}, 45^{\circ}, 60^{\circ}, 90^{\circ}\right.$, and $\left.120^{\circ}\right)$ theoretically.

The theoretical linear movement analysis for TWOMR at all angles $\theta=30^{\circ}, 45^{\circ}, 60^{\circ}, 90^{\circ}$, and $120^{\circ}$ is shown in Figures 11 , $12,13,14$, and 15 for forward $\left(V_{f}\right)$ direction and the theoretical linear movement analysis for the reverse $\left(V_{r}\right)$ direction is shown in Figures 17, 18, 19, 20, and 21. The path followed by TWOMR theoretically is termed as the "ideal path" in this paper.

\section{Experimental Tests}

Experimental tests are conducted at each angle $\left(\theta=30^{\circ}, 45^{\circ}\right.$, $60^{\circ}, 90^{\circ}$, and $120^{\circ}$ ) in forward and reverse direction. The experimental result shows that the TWOMR is deflecting or deviating from its "ideal path." These deflections are noted and plotted in graphs. Figures 8(a), 8(b), 8(c), 8(d), and 8(e) show the line diagram of the front and back movement analysis at $30^{\circ}, 45^{\circ}, 60^{\circ}, 90^{\circ}$, and $120^{\circ}$.

Figures 9(a), 9(b), 9(c), 9(d), and 9(e) show the real-time pictures of the angle between the wheels at $30^{\circ}, 45^{\circ}, 60^{\circ}, 90^{\circ}$, and $120^{\circ}$.

5.1. Forward Movement Analysis of TWOMR. For the forward movement analysis of TWOMR, two trials are conducted for each angle $\left(\theta=30^{\circ}, 45^{\circ}, 60^{\circ}, 90^{\circ}\right.$, and $\left.120^{\circ}\right)$ and deflection readings are plotted in Figures 11, 12, 13, 14, and 15, respectively.

The forward movement analysis shows that the deflections are minimum at angles $\theta=120^{\circ}$ and $60^{\circ}$, which 


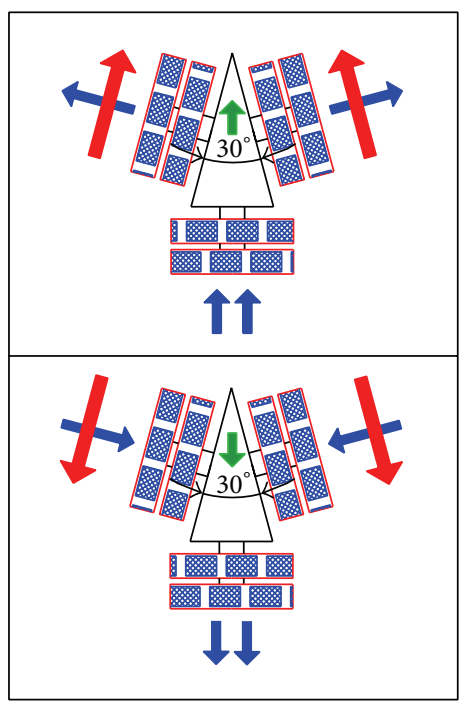

(a)

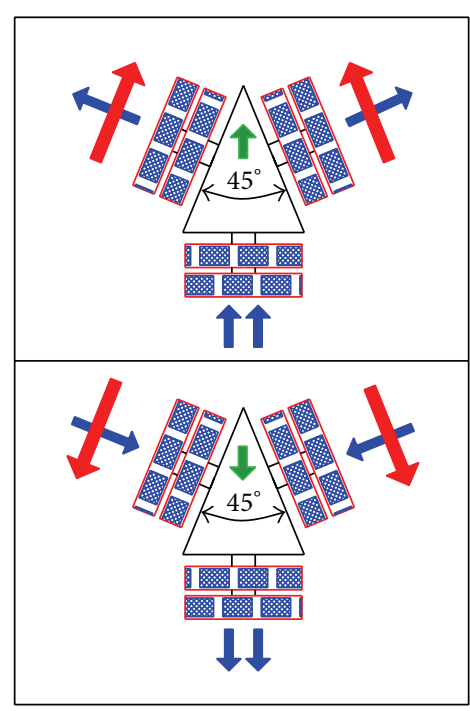

(b)

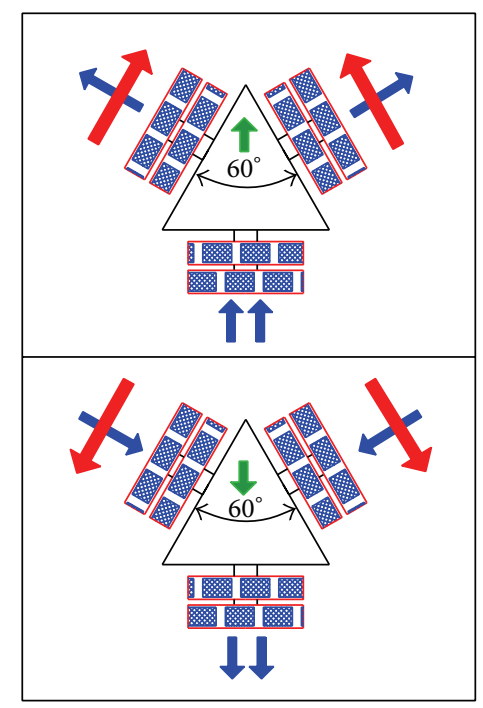

(c)

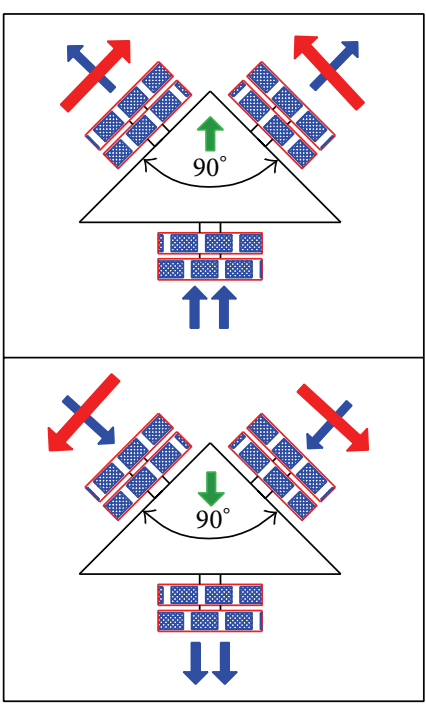

(d)

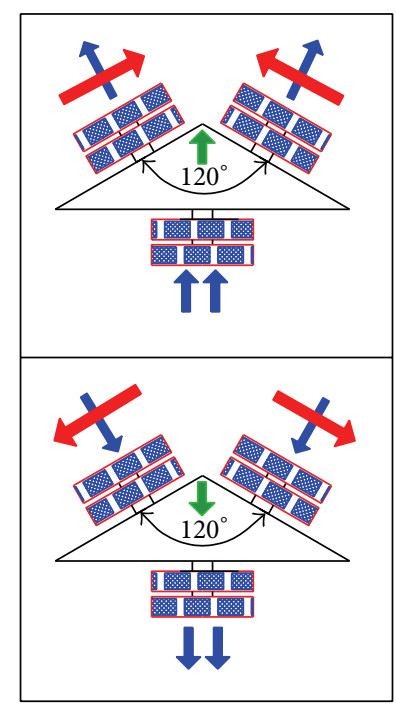

(e)

Figure 8: Front and back movement of TWOMR, wheel and roller rotations, when (a) $\theta=30^{\circ}$, (b) $\theta=45^{\circ}$, (c) $\theta=60^{\circ}$, (d) $\theta=90^{\circ}$, and (e) $\theta$ $=120^{\circ}$.

are almost negligible. Figure 10 shows one of the real-time experiments of the forward movement analysis of TWOMR at $\theta=45^{\circ}$. It was moved up to 3 metres and the deflections from the reference line were marked and analysed.

5.2. Reverse Movement Analysis of TWOMR. For the reverse movement analysis of TWOMR, two trials are conducted for each angle $\left(\theta=30^{\circ}, 45^{\circ}, 60^{\circ}, 90^{\circ}\right.$, and $\left.120^{\circ}\right)$ and deflection readings are plotted in Figures 17, 18, 19, 20, and 21, respectively.

The reverse movement analysis also shows that the deflections are minimum at angles $\theta=120^{\circ}$ and $60^{\circ}$, which are almost negligible.

Hence, the deflections are arising at almost every angle $(\theta)$. And these deflections are minimum and almost negligible at angles $\theta=120^{\circ}$ and $60^{\circ}$, in both forward and reverse direction. Hence, these two angles are optimum angles for the forward and reverse movement of TWOMR and its performance is also very efficient. One of the real-time experiments of the reverse movement analysis of TWOMR at $\theta=45^{\circ}$ is shown in Figure 16. It was moved up to 3 metres and the deflections from the reference line were marked and analysed.

\section{Discussions and Conclusions}

A practical implementation of an omnidirectional mobile robot was the main focus of this work. A unique device of Angle Variable Chassis (AVC) was designed, and a three-wheeled omnidirectional mobile robot (TWOMR) was implemented and its kinematics model was described. A prototype of TWOMR was practically run on the cement 


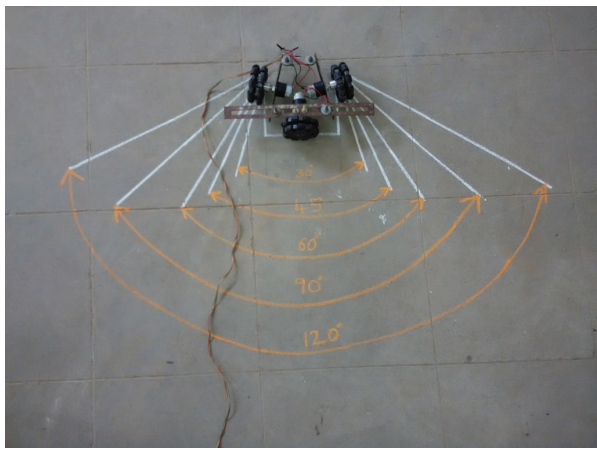

(a)

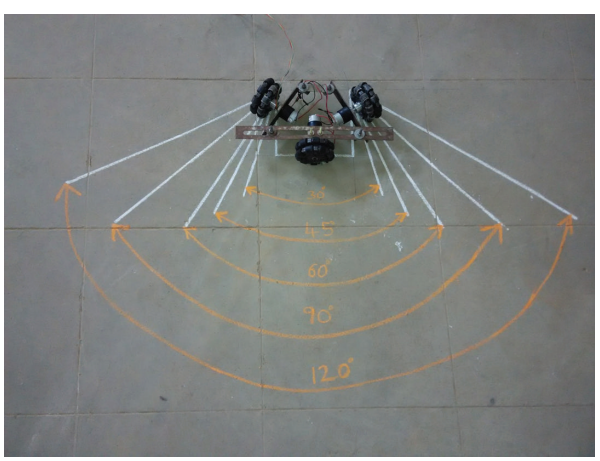

(c)

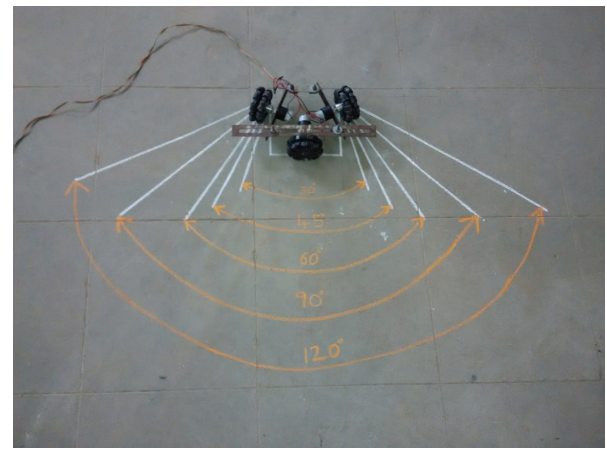

(b)

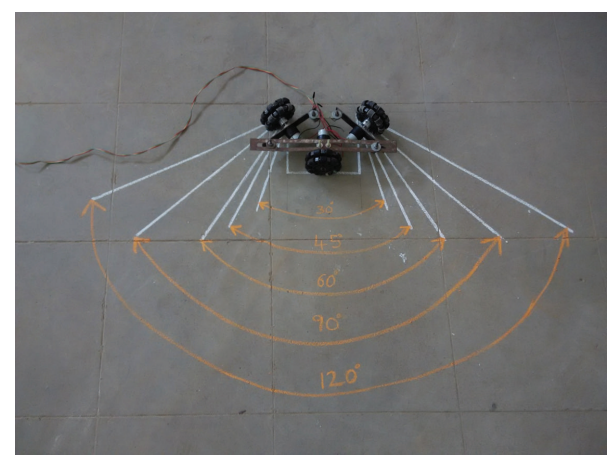

(d)

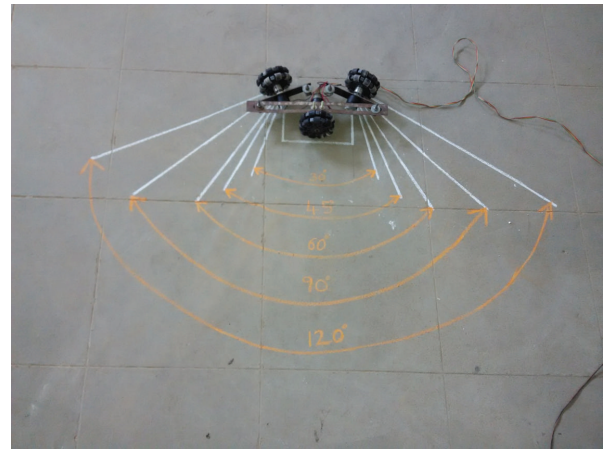

(e)

Figure 9: Three-wheeled omnidirectional mobile robot (TWOMR). (a) $\theta=30^{\circ}$, (b) $\theta=45^{\circ}$, (c) $\theta=60^{\circ}$, (d) $\theta=90^{\circ}$, and (e) $\theta=120^{\circ}$.

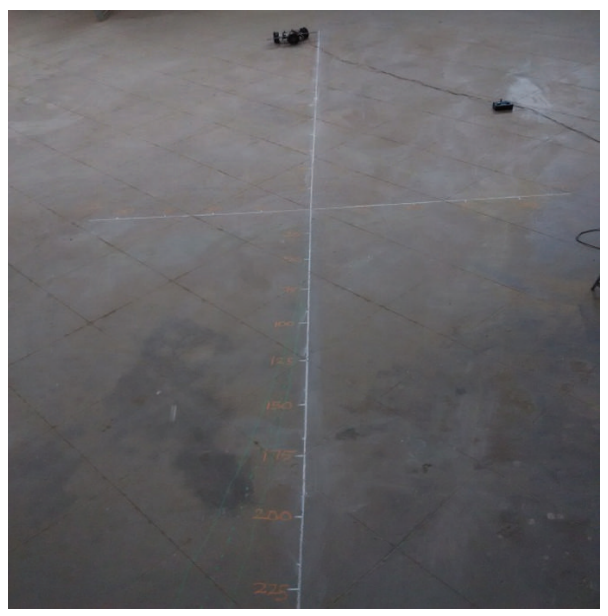

FIGURE 10: Real-time experiment of forward movement analysis of TWOMR at $\theta=45^{\circ}$. 


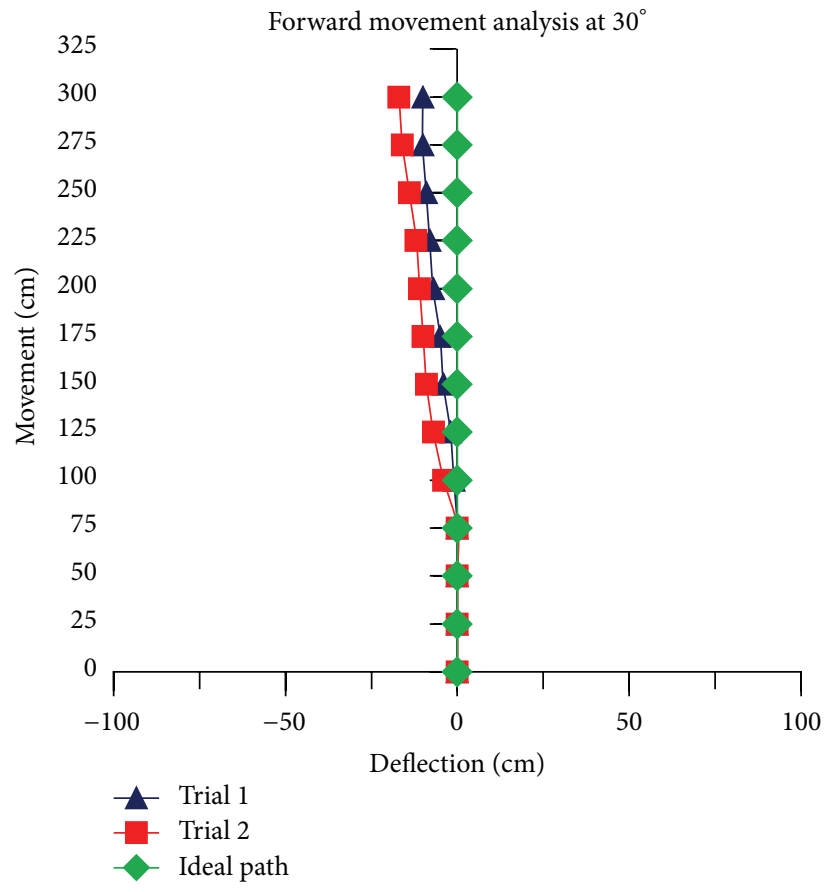

FIGURE 11: Forward movement versus deflections at $\theta=30^{\circ}$.

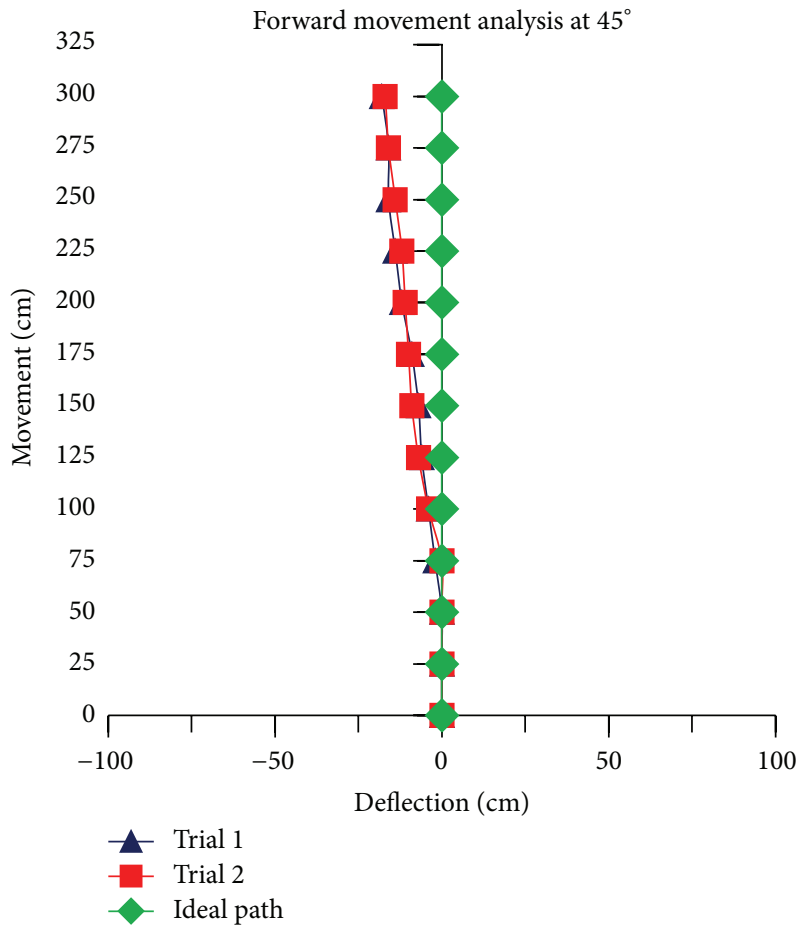

FIgURE 12: Forward movement versus deflections at $\theta=45^{\circ}$.

floor and several experimental results indicated that the omnidirectional mobile robot had a desirable full mobility and smooth motion.

There are some errors originated from several sources such as the nonsteady condition in omnidirectional wheels

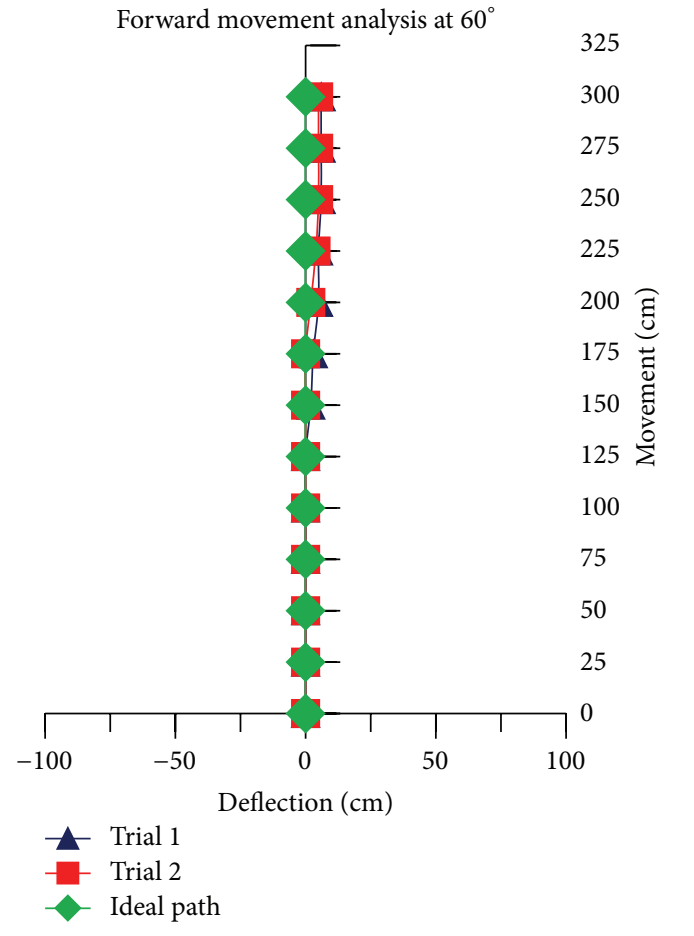

FIGURE 13: Forward movement versus deflections at $\theta=60^{\circ}$.

during motion, the effect of friction in working model, and the effect of omniwheel rollers on omniwheel. Irrespective of these errors, TWOMR showed effective movement at angles $120^{\circ}$ and $60^{\circ}$. Hence, these two angles are optimum angles for the movement of TWOMR, as the deflections are 


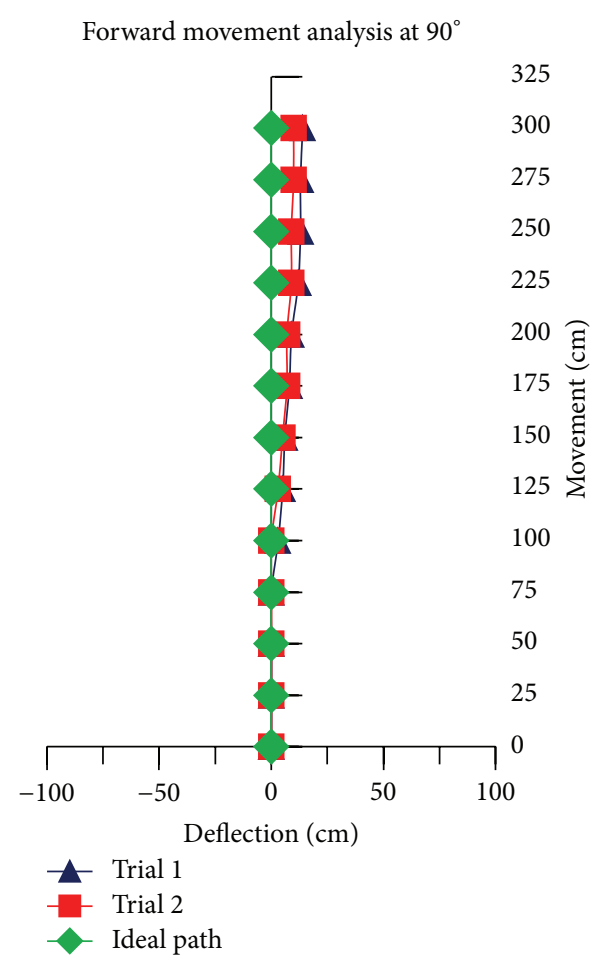

Figure 14: Forward movement versus deflections at $\theta=90^{\circ}$.

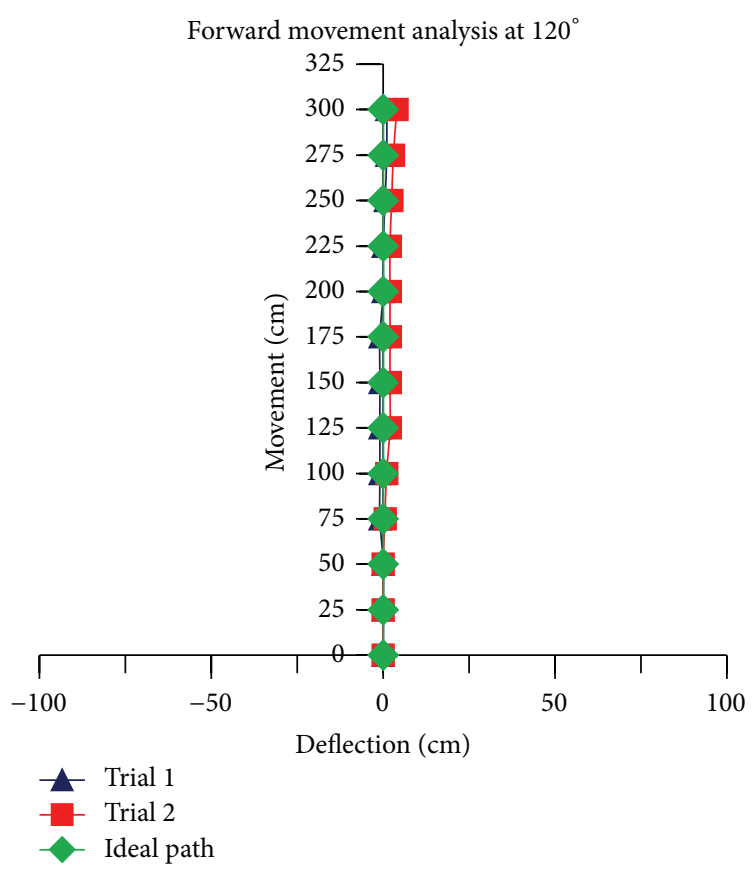

FIGURE 15: Forward movement versus deflections at $\theta=120^{\circ}$. 


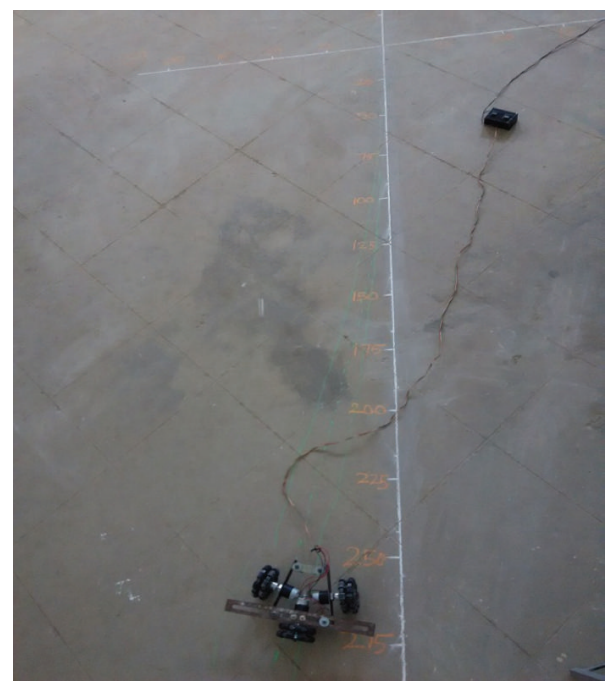

FIGURE 16: Real-time experiment of reverse movement analysis of TWOMR at $\theta=45^{\circ}$.

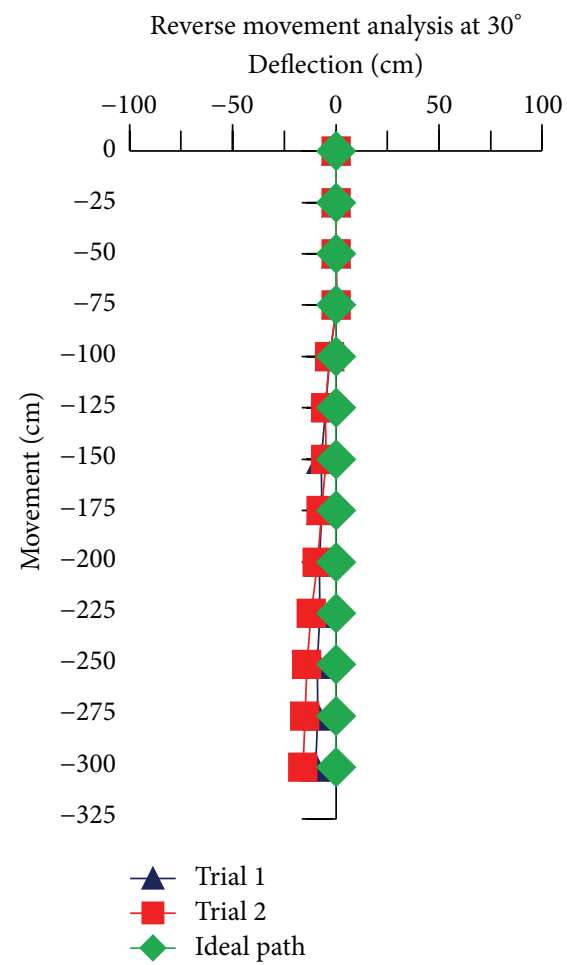

FIGURE 17: Reverse movement versus deflections at $\theta=30^{\circ}$.

minimum and negligible. The TWOMR has greater speed at $60^{\circ}$ compared to $120^{\circ}$. Hence, $60^{\circ}$ is most preferred for the works to be done quickly. These TWOMRs are suitable and applicable in congested environments and radiation environments where humans cannot work.

Omnidirectional mobile robot can move in all directions without steering the wheels and it can rotate about its axis.

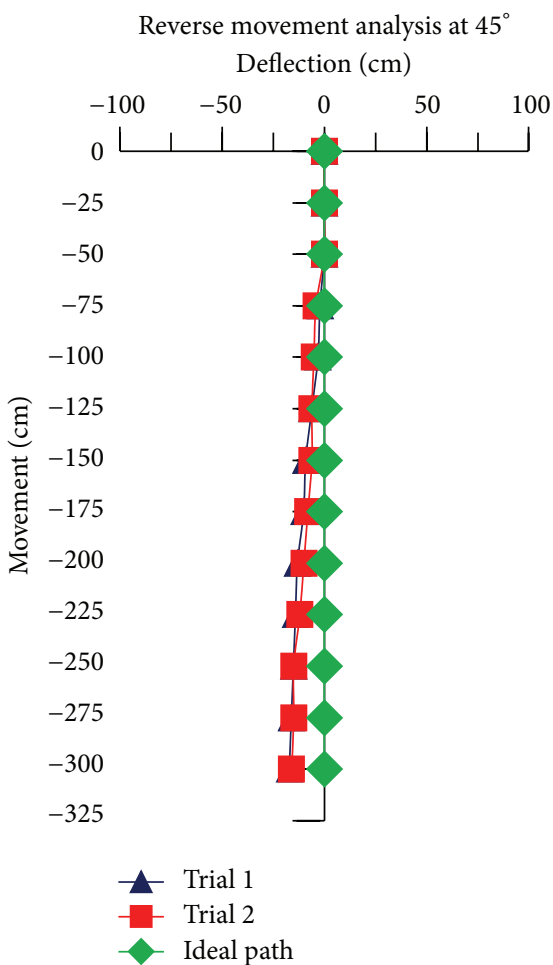

FIGURE 18: Reverse movement versus deflections at $\theta=45^{\circ}$.

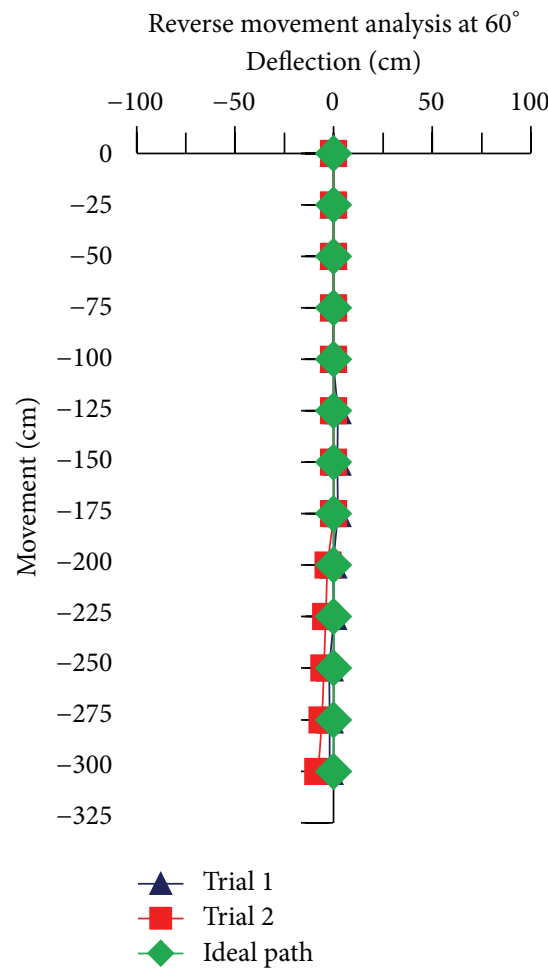

FIGURE 19: Reverse movement versus deflections at $\theta=60^{\circ}$. 


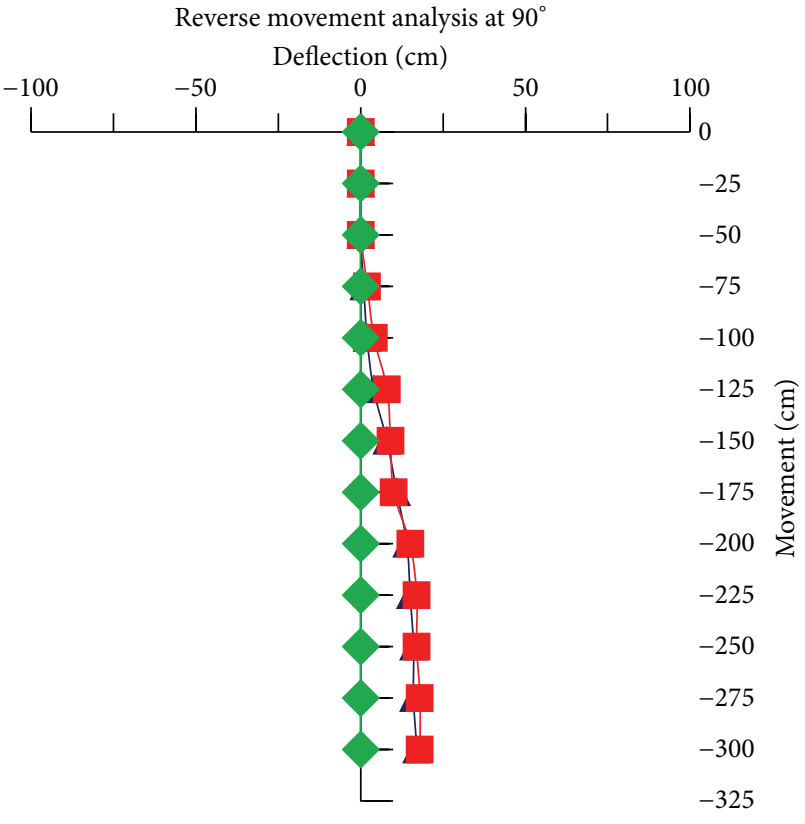

$\boldsymbol{\Delta}$ Trial 1

Trial 2

Ideal path

FIgURE 20: Reverse movement versus deflections at $\theta=90^{\circ}$.

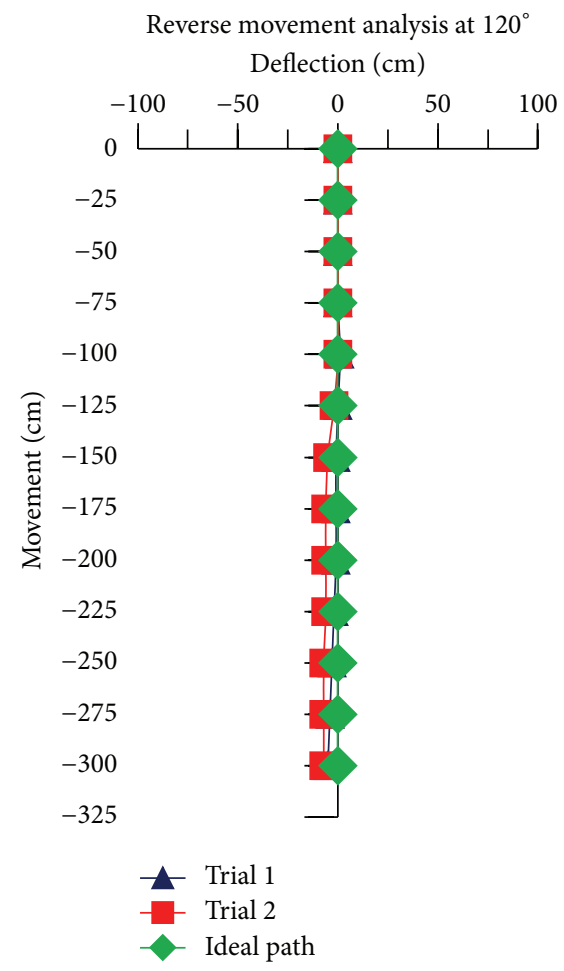

FIGURE 21: Reverse movement versus deflections at $\theta=120^{\circ}$.
In this paper, for experimental purpose, only the linear movement analysis has been done. However, there are minimum and negligible deflections arising. Hence, the control of the deflections, movement analysis on various surfaces, nonlinear movement analysis, and the ideal movement of the TWOMR will be done in future.

\section{Conflict of Interests}

The authors declare that there is no conflict of interests regarding the publication of this paper.

\section{References}

[1] Y.-C. Lee, D. V. Lee, J. H. Chung, D. A. Bennett, and S. A. Velinsky, "Design and control of the ball wheel drive mechanism for a robust omnidirectional wheeled mobile platform," in Romansy 16, vol. 487 of CISM Courses and Lectures, pp. 387-394, Springer, 2006.

[2] I. Doroftei, V. Grosu, and V. Spinu, "Design and Control of an Omni-Directional Mobile Robot," in Novel Algorithms and Techniques in Telecommunications, Automation and Industrial Electronics, pp. 105-110, Springer, Dordrecht, The Netherlands, 2008.

[3] Y. Guorong and Z. Haibing, "A new kind of wheel-model alldirectional moving mechanism," Journal of Harbin Institute of Technology, vol. 33, no. 6, 2001.

[4] Z. Chao, Research on the design and control of a robot Omnidirectional platform [M.S. thesis], Hohai University, Nanjing, China, 2007.

[5] Y. Hirata, Y. Koike, Z. Liu, and K. Kosuge, "Development of omni-directional mobile base with servo brakes for passive dance partner robot," in Proceedings of the IEEE International Conference on Robotics and Biomimetics (ROBIO '08), pp. 676681, Bangkok, Thailand, February 2009.

[6] M. Endo, K. Hirose, Y. Sugahara et al., "Trajectory generation for multiple robots of a car transportation system," in Distributed Autonomous Robotic Systems 8, pp. 305-314, Springer, Berlin, Germany, 2009.

[7] C. Ye, S. Ma, and L. Hui, "An omnidirectional mobile robot," Science China Information Sciences, vol. 54, no. 12, pp. 26312638, 2011.

[8] S. Kim, I. Jeong, and S. Lee, "Systematic isotropy analysis of a mobile robot with three active caster wheels," in Advanced Intelligent Computing Theories and Applications. With Aspects of Theoretical and Methodological Issues: Third International Conference on Intelligent Computing, ICIC 2007 Qingdao, China, August 21-24, 2007 Proceedings, vol. 4681 of Lecture Notes in Computer Science, pp. 587-597, Springer, Berlin, Germany, 2007.

[9] S.-B. Huang, D.-S. Chen, and H.-Q. Gong, "Research on the impact of the number of wheels and layouts to the performances of Omni-directional chassis," in Intelligent Robotics and Applications: 5th International Conference, ICIRA 2012, Montreal, QC, Canada, October 3-5, 2012, Proceedings, Part III, vol. 7508 of Lecture Notes in Computer Science, pp. 258-267, Springer, Berlin, Germany, 2012.

[10] E.-J. Jung, B.-J. Yi, and W. K. Kim, "Motion planning algorithms of an omni-directional mobile robot with active caster wheels," Intelligent Service Robotics, vol. 4, no. 3, pp. 167-180, 2011. 
[11] J.-T. Zou, F.-C. Chiang, and K. L. Su, "The study of path error for an omnidirectional home care mobile robot," Artificial Life and Robotics, vol. 16, no. 2, pp. 127-131, 2011.

[12] H. Asama, M. Sato, L. Bogoni, H. Kaetsu, A. Matsumoto, and I. Endo, "Development of an omni-directional mobile robot with 3 DOF decoupling drive mechanism," in Proceedings of the IEEE International Conference on Robotics and Automation, vol. 2, pp. 1925-1930, Nagoya, Japan, May 1995.

[13] B.-J. Yi and W. K. Kim, "The kinematics for redundantly actuated omnidirectional mobile robots," Journal of Robotic Systems, vol. 19, no. 6, pp. 255-267, 2002.

[14] M. Wada, A. Takagi, and S. Mori, "Caster drive mechanisms for holonomic and omnidirectional mobile platforms with no over constraint," in Proceedings of the IEEE International Conference on Robotics and Automation (ICRA '00), vol. 2, pp. 1531-1538, San Francisco, Calif, USA, April 2000.

[15] N. Ushimi, M. Yamamoto, and A. Mohri, "Two wheels caster type odometer for omni-directional vehicles," in Proceedings of the IEEE International Conference on Robotics and Automation, pp. 497-502, Taipei, Taiwan, September 2003.

[16] S. K. Saha, J. Angeles, and J. Darcovich, "The design of kinematically isotropic rolling robots with omnidirectional wheels," Mechanism and Machine Theory, vol. 30, no. 8, pp. 1127-1137, 1995.

[17] J. A. Batlle and A. Barjau, "Holonomy in mobile robots," Robotics and Autonomous Systems, vol. 57, no. 4, pp. 433-440, 2009.

[18] A. P. Mohanraj, A. Elango, D. Ragavendhiran, P. V. Raja, and K. Ashok, "Design and movement analysis of single roller Omni directional wheeled robot for different assembly structures," Applied Mechanics and Materials, vol. 592-594, pp. 2324-2328, 2014.

[19] J. E. M. Salih, M. Rizon, and S. Yaacob, "Designing omnidirectional mobile robot with mecanum wheel," American Journal of Applied Sciences, vol. 3, no. 5, pp. 1831-1835, 2006.

[20] Y. P. Leow, K. H. Low, and W. K. Loh, "Kinematic modelling and analysis of mobile robots with omni-directional wheels," in Proceedings of the 7th International Conference on Control, Automation, Robotics and Vision (ICARCV '02), vol. 2, pp. 820825, IEEE, Singapore, December 2002. 


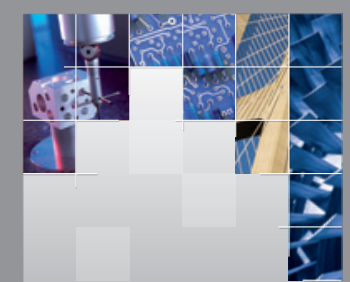

\section{Enfincering}
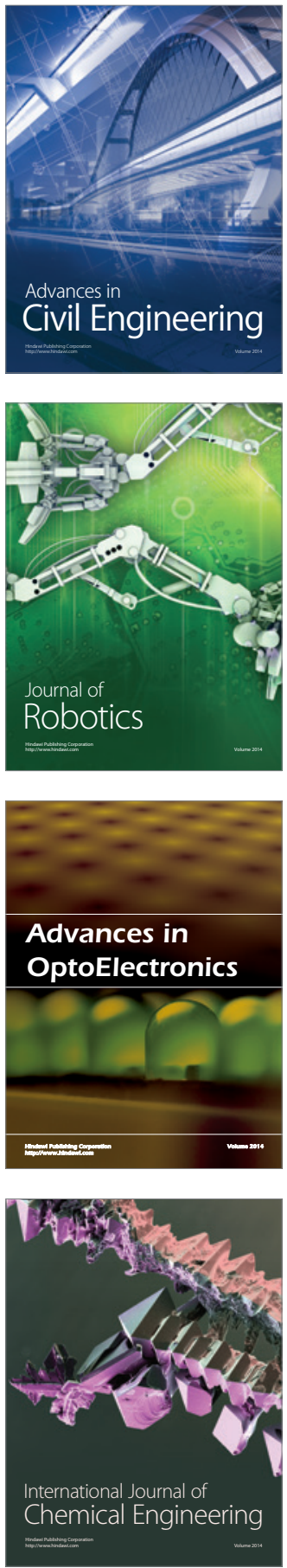

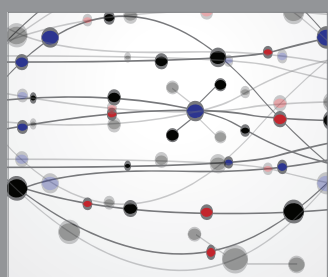

The Scientific World Journal

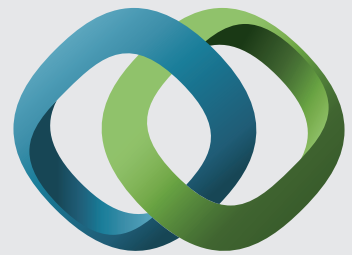

\section{Hindawi}

Submit your manuscripts at

http://www.hindawi.com
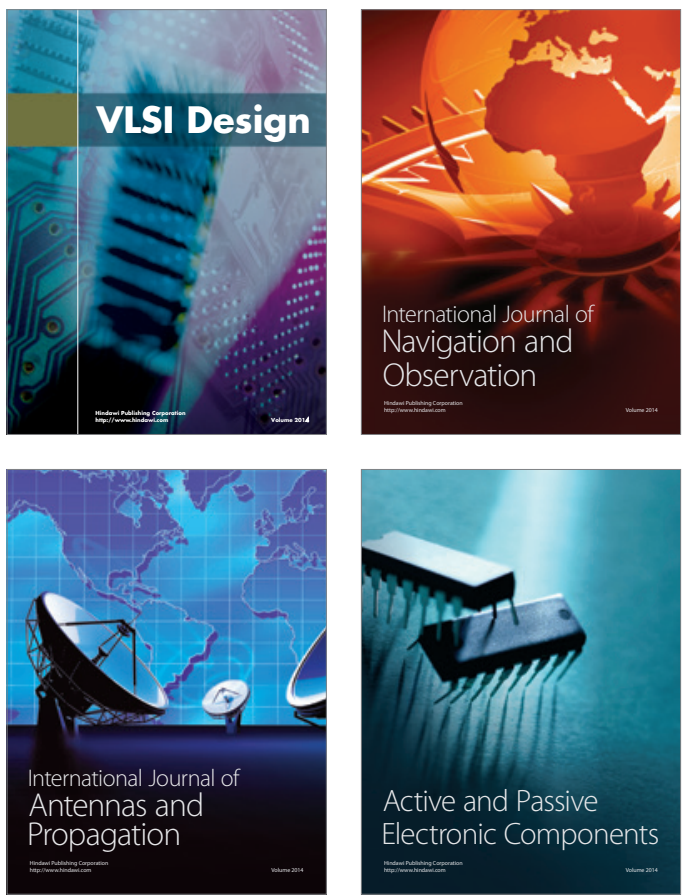
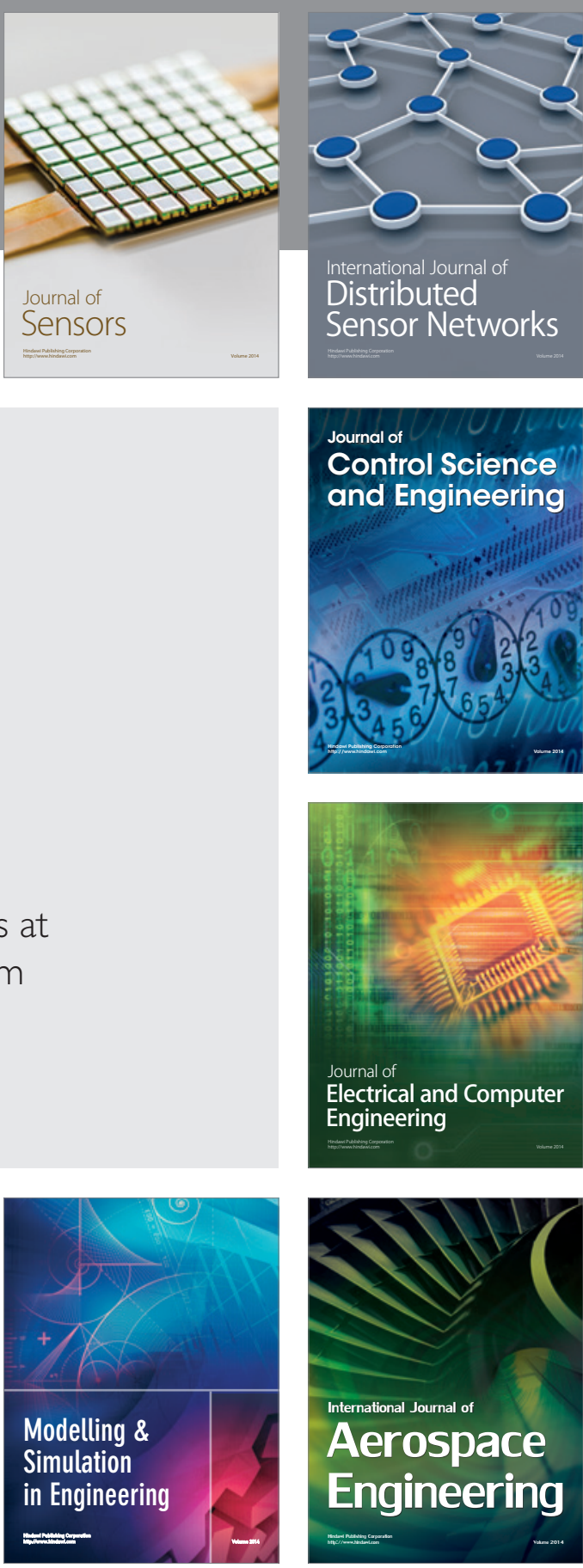

International Journal of

Distributed

Sensor Networks

Journal of

Control Science

and Engineering
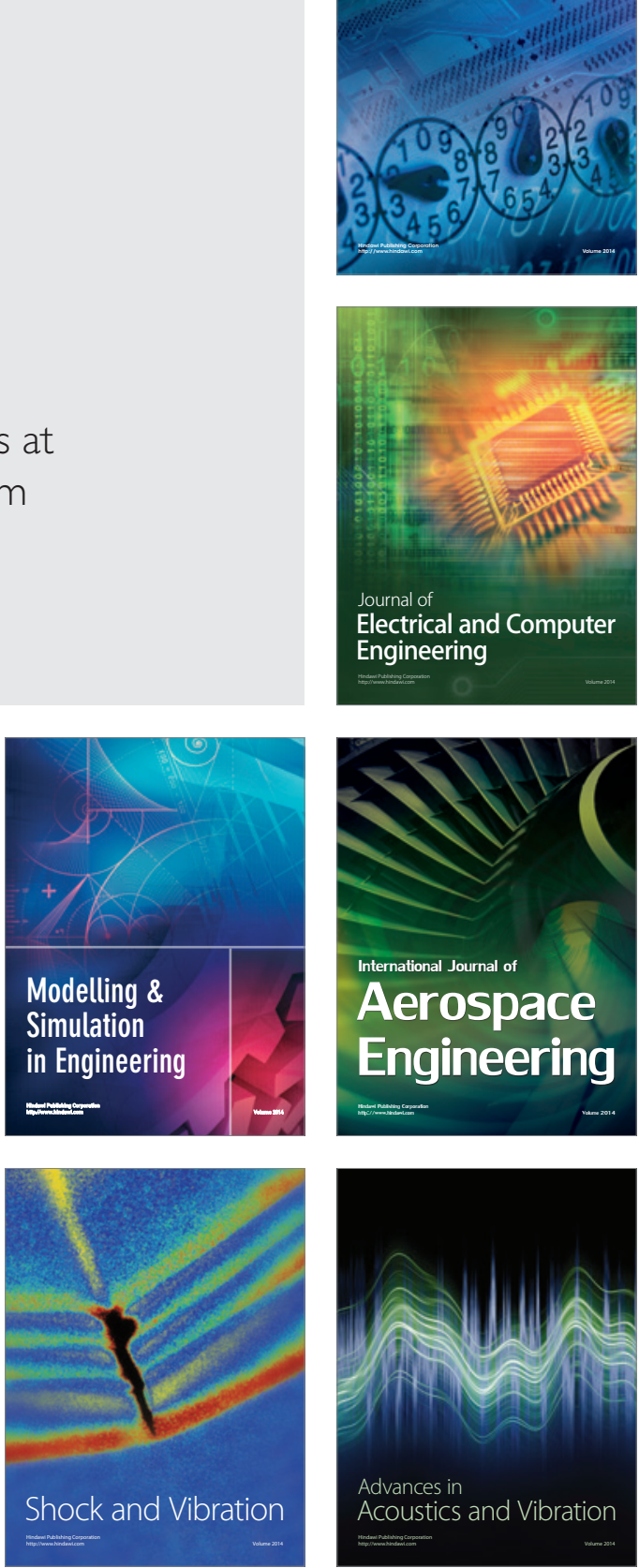